\title{
RF plasma simulations using the TOMATOR 1D code: a case study for TCV helium ECRH plasmas
}

\author{
T. Wauters ${ }^{1}$, J. Buermans ${ }^{1}$, R. Haelterman1 ${ }^{1,2}$, V. Moiseenko ${ }^{3}$, D. Ricci ${ }^{4}$, T. \\ Verhaeghe $^{1,2}$, S. Coda ${ }^{5}$, D. Douai ${ }^{6}$, A. Hakola ${ }^{7}$, A. Lyssoivan ${ }^{1}$, D. Van Eester ${ }^{1}$, the \\ TCV team*, the EUROfusion MST1 team ${ }^{* *}$
}

${ }^{1}$ Laboratory for Plasma Physics, LPP-ERM/KMS, B-1000 Brussels, Belgium, Trilateral Euregio Cluster (TEC) Partner.

${ }^{2}$ Departement of Mathematics, ERM/KMS, B-1000 Brussels, Belgium.

${ }^{3}$ Institute of Plasma Physics of the NSC KIPT, Kharkov, Ukraine

${ }^{4}$ Istituto di Fisica del Plasma CNR, I-20125 Milano, Italy

${ }^{5}$ Ecole Polytechnique Fédérale de Lausanne (EPFL), Swiss Plasma Center (SPC), CH-1015 Lausanne, Switzerland

${ }^{6}$ CEA, IRFM, F-13108 Saint Paul Lez Durance, France

${ }^{7}$ VTT Technical Research Centre of Finland, PO Box 1000, FIN-02044 VTT, Finland

${ }^{*}$ See author list of S. Coda et al 2019 Nucl. Fusion 59112023 (https://doi.org/10.1088/1741-

$4326 / \mathrm{ab} 25 \mathrm{cb})$.

** See author list of B. Labit et al 2019 Nucl. Fusion 59086020 (https://doi.org/10.1088/17414326/ab2211)

E-mail: t.wauters@fz-juelich.de

Received xxxxxx

Accepted for publication $\mathrm{xxxxxx}$

Published xxxxxx

\begin{abstract}
The 1-dimensional reaction-diffusion-convection code TOMATOR-1D describes plasma production by RF waves inside a tokamak using the Braginskii continuity and heat balance equations. The model simulates self-consistent radial density and temperature profiles for magnetised plasma mixtures of hydrogen and helium. The model reproduces the density profiles of X2 ECRH plasmas on TCV and proposes a Bohm-like poloidal magnetic field dependent scaling for anomalous diffusion and a convection scaling that results from drifts in the toroidal magnetic field configuration. A relation is proposed between the anomalous diffusion and the outward convection in toroidal plasmas. It is found that the EC absorption efficiency decreases at higher power, which is understood from the acceleration of electrons beyond the optimal energy for the electron impact ionisation of helium. A dramatic increase of the absorption efficiency is seen at intermediate vertical magnetic field values of $B_{z}=$ $0.25-0.5 \% B_{T}$ which results in the highest density plasmas. Losses along the field lines in the vertical direction become dominant at higher fields which effectively reduces the plasma density in these discharges. To arrive at predictive capabilities towards ECRH plasmas on JT60SA and ITER, the proposed scalings, subsuming dependencies on the torus major radius and the toroidal field strength, need to be validated in a multi-machine study.
\end{abstract}

Keywords: ECWC, ECRH, wall conditioning, plasma start-up, tokamak, TCV 


\section{Introduction}

Radio-frequency and microwave (RF) plasmas find applications in tokamaks for wall conditioning and breakdown assistance. The currentless discharges, without equilibrium and typically partially ionised, are produced by resonant or collisional absorption of RF power. Predicting the RF plasma parameters for future large devices such as JT-60SA and ITER requires a good understanding of the power absorption and transport mechanisms of the toroidal magnetised plasmas. The 1D transport oriented model for magnetized toroidal RF discharges, TOMATOR-1D, is developed to provide insight in these phenomena. Its robust mathematical implementation with an unconditionally stable temporal discretisation, using the fully implicit backward differentiation scheme of second order complemented by an adaptive time stepping scheme, provides a practical means to benchmark confinement scalings or power absorption modules against experimental data sets.

The first part of this paper describes the model starting from the 1D transport equations. The implemented scalings for the diffusion and convection coefficients are discussed, as well as the losses along the magnetic field lines. A summary of the included elementary collisions is given and finally the edge conditions are discussed. The second part of the paper presents a case study where the model is benchmarked against data from helium ECRH (electron cyclotron resonance heating) plasmas on TCV. By reproducing the measured radial density profiles, insight is obtained in the dependencies of the transport coefficients and power absorption efficiency on the applied vertical field or the launched ECRH power.

\section{Model description}

To describe numerically in a 1D approach the evolution of RF plasma parameters in tokamaks, the standard continuity and heat balance equations given by Braginskii [1] are adopted in cylindrical slab geometry. The diffusion-convectionreaction equation is solved for both density $n(r, t)$ and energy density $E(r, t)=3 / 2 n(r, t) T(r, t)$ for each of the plasma ions and neutrals, with $T(r, t)$ their respective Maxwellian temperatures:

$$
\begin{gathered}
\dot{n}(r, t)=\frac{1}{r} \frac{\partial}{\partial r} r D(r, t) \frac{\partial}{\partial r} n(r, t)-\frac{1}{r} \frac{\partial}{\partial r} r V(r, t) n(r, t) \\
\quad+S(r, t) \\
\dot{E}(r, t)=\frac{1}{r} \frac{\partial}{\partial r} r \gamma_{D} D(r, t) \frac{\partial}{\partial r} E(r, t) \\
\quad-\frac{1}{r} \frac{\partial}{\partial r} r V(r, t) E(r, t) \\
+S_{E}(r, t)
\end{gathered}
$$

Both the diffusion coefficient $D(r, t)$ and the convection velocity $V(r, t)$ are implemented such that they can depend on the radial coordinate $r$. The source terms, $S(r, t)$ and $S_{E}(r, t)$, include the particle and energy changes by collisional processes, external particle sources such as neutral gas injection, toroidal losses on limiters and losses across or along the field lines in the vertical direction. The factor $\gamma_{D}$ in equation (2) correct the energy transport for the typical $(\sim 3)$ times poorer energy confinement compared to the particle confinement.

The equations are implemented for hydrogen-helium plasmas $\left(\mathrm{H}_{2}, \mathrm{H}, \mathrm{H}^{+}, \mathrm{H}_{2}^{+}, \mathrm{H}_{3}^{+}, \mathrm{He}, \mathrm{He}^{+}, \mathrm{He}^{2+}\right)$. The electron transport is modelled as the sum of the ion transport, assuming charge neutrality. To cope with the sharp density and energy gradients at the edges of the simulation domain or at RF resonances, the density and energy profiles in the model are discretized by hermitian cubic shape functions. These are defined by their value and derivative at the grid points. Piecewise linear functions are used for the diffusion, convection and source functions.

\subsection{Elementary processes in hydrogen-helium plasmas}

Elementary atomic and molecular collision processes are evaluated at each grid point and time instance in the model. Radiative-collisional models are applied where possible to obtain effective collision rate coefficients, avoiding treating excited states as separate species [2], [3]. Table 1 summarises the included inelastic collisions, adapted from [4], and grouped into (i) electron collisions with $\mathrm{H}_{\text {and }} \mathrm{H}^{+}$(1-4), (ii) electron collisions with $\mathrm{H}_{2}, \mathrm{H}_{2}^{+}$and $\mathrm{H}_{3}^{+}$(5-13), (iii) electron collisions with $\mathrm{He}, \mathrm{He}^{+}$and $\mathrm{He}^{2+}$ (14-17), charge exchange (18-25) and other ion impact reactions (26-33). Coulomb collisions between the charged particles as well as elastic ionneutral and neutral-neutral collisions, especially important for temperatures below $10 \mathrm{eV} \mathrm{[5]-[7]} \mathrm{have} \mathrm{been} \mathrm{adopted} \mathrm{from} \mathrm{[4].}$ While electron cooling on hydrogenic species is included through the considered collisional reactions, total cooling rates are implemented for electron collisions with $\mathrm{He}, \mathrm{He}^{+}$and $\mathrm{He}^{2+}[8]$.

Table 1: Summary of the included elementary inelastic processes in hydrogen-helium plasmas

\begin{tabular}{ccc}
\hline & Collisional reaction & Ref. \\
\hline & Electron collisions with $\mathrm{H}$ and $\mathrm{H}^{+}$ & \\
1 & $\mathrm{e}+\mathrm{H} \rightarrow \mathrm{e}+\mathrm{H}^{*}$ & {$[4]$} \\
2 & $\mathrm{e}+\mathrm{H} \rightarrow \mathrm{e}+\mathrm{H}^{+}+\mathrm{e}$ & {$[4]$} \\
3 & $\mathrm{e}+\mathrm{H}^{+}+\mathrm{e} \rightarrow \mathrm{e}+\mathrm{H}$ & {$[4]$} \\
4 & $\mathrm{e}+\mathrm{H}^{+} \rightarrow \mathrm{H}+h v$ & {$[4]$} \\
\hline & Electron collisions with $\mathrm{H}_{2}, \mathrm{H}_{2}^{+}$and $\mathrm{H}_{3}^{+}$ & \\
5 & $\mathrm{e}+\mathrm{H}_{2} \rightarrow \mathrm{e}+\mathrm{H}_{2}^{*}$ & {$[9]$} \\
6 & $\mathrm{e}+\mathrm{H}_{2} \rightarrow \mathrm{e}+\mathrm{H}+\mathrm{H}$ & {$[4]$} \\
7 & $\mathrm{e}+\mathrm{H}_{2} \rightarrow \mathrm{e}+\mathrm{H}_{2}^{+}+\mathrm{e}$ & {$[4]$} \\
8 & $\mathrm{e}+\mathrm{H}_{2} \rightarrow \mathrm{e}+\mathrm{H}+\mathrm{H}^{+}+\mathrm{e}$ & {$[9]$} \\
9 & $\mathrm{e}+\mathrm{H}_{2}^{+} \rightarrow \mathrm{H}_{2}+h v$ & {$[4]$} \\
10 & $\mathrm{e}+\mathrm{H}_{2}^{+} \rightarrow \mathrm{e}+\mathrm{H}^{+}+\mathrm{H}^{+}$ & {$[9]$} \\
11 & $\mathrm{e}+\mathrm{H}_{2}^{+} \rightarrow \mathrm{H}+\mathrm{H}^{*}$ & {$[9]$} \\
& $\mathrm{e}+\mathrm{H}_{2}^{+} \rightarrow \mathrm{H}+\mathrm{H}^{*}$ & {$[9]$}
\end{tabular}


12

13

Electron collisions with $\mathrm{He}, \mathrm{He}^{+}$and $\mathrm{He}^{2+}$

\begin{tabular}{ccc}
14 & $\mathrm{e}+\mathrm{He} \rightarrow \mathrm{e}+\mathrm{He}^{+}+\mathrm{e}$ & {$[4]$} \\
15 & $\mathrm{e}+\mathrm{He}^{+} \rightarrow \mathrm{He}+h v$ & {$[4]$} \\
16 & $\mathrm{e}+\mathrm{He}^{+} \rightarrow \mathrm{e}+\mathrm{He}^{2+}+\mathrm{e}$ & {$[4]$} \\
17 & $\mathrm{e}+\mathrm{He}^{2+} \rightarrow \mathrm{He}^{+}+h v$ & {$[4]$} \\
\hline & Ion impact reactions & \\
18 & $\mathrm{CX}: \mathrm{H}^{+}+\mathrm{H}$ & {$[10]$} \\
19 & $\mathrm{CX}: \mathrm{H}^{+}+\mathrm{H}_{2}$ & {$[9]$} \\
20 & $\mathrm{CX}: \mathrm{H}_{2}^{+}+\mathrm{H}_{2}$ & {$[9]$} \\
21 & $\mathrm{CX}: \mathrm{He}^{+}+\mathrm{H}$ & {$[11]$} \\
22 & $\mathrm{CX}: \mathrm{He}^{+}+\mathrm{He}$ & {$[9]$} \\
23 & $\mathrm{CX}: \mathrm{He}^{2+}+\mathrm{H}$ & {$[12]$} \\
24 & $\mathrm{CX}: \mathrm{He}^{2+}+\mathrm{He}^{+} \mathrm{He}+\mathrm{He}^{+}$ & {$[13]$} \\
25 & $\mathrm{CX}: \mathrm{He}^{2+}+\mathrm{He}^{+} \rightarrow \mathrm{He}^{+} \mathrm{He}$ & {$[9]$} \\
26 & $\mathrm{H}_{2}^{+}+\mathrm{H}_{2} \rightarrow \mathrm{H}_{3}^{+}+\mathrm{H}$ & {$[9]$} \\
27 & $\mathrm{H}^{+}+\mathrm{H}^{+} \rightarrow \mathrm{H}^{+}+\mathrm{H}^{*}$ & {$[9]$} \\
28 & $\mathrm{H}^{+}+\mathrm{H}_{2} \rightarrow \mathrm{H}^{+}+\mathrm{H}_{2}^{*}$ & {$[9]$} \\
29 & $\mathrm{H}^{+}+\mathrm{H} \rightarrow \mathrm{H}^{+}+\mathrm{H}^{+}+\mathrm{e}$ & {$[9]$} \\
30 & $\mathrm{H}^{+}+\mathrm{He}^{+} \rightarrow \mathrm{H}^{+}+\mathrm{He}^{+}+\mathrm{e}$ & {$[9]$} \\
31 & $\mathrm{H}^{+}+\mathrm{H}_{2} \rightarrow \mathrm{H}^{+}+\mathrm{H}_{2}^{+}+\mathrm{e}$ & {$[9]$} \\
32 & $\mathrm{H}^{+}+\mathrm{H}_{2}^{+} \rightarrow \mathrm{H}^{+}+\mathrm{H}^{+}+\mathrm{H}$ & {$[9]$} \\
33 & $\mathrm{He}^{+}+\mathrm{H}_{2} \rightarrow \mathrm{He}^{+}+\mathrm{H}^{+}+\mathrm{H}$ & {$[9]$} \\
\hline
\end{tabular}

\subsection{Charged particle transport and neutrals}

2.2.1 Diffusion. Yoshikawa described the anomalous diffusion of a plasma across a magnetic field [14]. The diffusion results from turbulence in the plasma and is inversely proportional to the magnetic field. According to Bohm's formula, the diffusion of electrons across the magnetic field is given by

$$
D=\frac{1}{3} f_{D} v_{c, e} r_{e} \lambda_{e}
$$

where $v_{c, e}$ is the collision frequency of electrons with ions, $r_{e}$ is the Larmor radius of the electron at the mean thermal velocity and $\lambda_{e}$ is the electron mean free path. The numerical coefficient $f_{D}$ was taken as $1 / 16$ by Bohm and experimentally set at 0.21 by Spitzer [14]. In the case study described in section 3 , the radial diffusion $D_{r}$ is implemented as

$$
D_{r}=\frac{1}{3} f_{D} v_{c, i}\left(r_{i}+\lambda_{i} \frac{B_{r}}{B_{\varphi}}\right) \lambda_{i}
$$

where $v_{c, i}$ is the collision frequency of the ions, $r_{i}$ is the Larmor radius of the ion at the mean thermal velocity, $\lambda_{i}$ is the ion mean free path, while $B_{r}$ and $B_{\varphi}$ are the local radial and toroidal components of the vacuum magnetic field. The gyration radius in the equation above is increased by a distance that corresponds to the radial projection of the mean free path along the inclined field lines. Similarly, the vertical diffusion coefficient $D_{z}$ is implemented as

$$
D_{z}=\frac{1}{3} f_{D} v_{c, i}\left(r_{i}+\lambda_{i} \frac{B_{z}}{B_{\varphi}}\right) \lambda_{i}
$$

with $B_{z}$ the local vertical component of the vacuum magnetic field. The loss rate of charged particles by vertical diffusion is included by the characteristic time $\tau_{v}$ at the grid points

$$
\tau_{z}=\frac{b^{2}(r)}{2 D_{z}}
$$

with $b(r)$ half the height of the vessel at radial position $r$.

2.2.2 Convection. The charge dependent vertical drifts due to the curvature and radial gradient of the toroidal field $B_{\varphi}(r)$ results in a vertical electric field $E_{z}$ that drives the outward convection of the plasma column. The outward velocity depends on the vertical electric field and the toroidal field $B_{T}=B_{\varphi}(R)$, with $R$ being the major radius of the torus, as $E_{z} / B_{T}$. An experimental scaling for $E_{z}$ in $\mathrm{V} / \mathrm{m}$ is given by Nakao [15]:

$$
E_{z, 1}=5 \frac{T_{e}}{a}
$$

with $a$ the vessel minor radius in $\mathrm{cm}$ and $T_{e}$ the electron temperature in $\mathrm{eV}$. The electric field is expected to decrease upon applying a vertical magnetic field that allows shortcircuiting electron currents to flow along the inclined field lines. The strength of the resulting quasi steady-state electric field is derived by Müller [16] as

$$
E_{z, 2}=\frac{m_{e}}{q_{e}} \frac{v_{\|}^{e, i} v_{\nabla B}}{\sin ^{2} \theta}
$$

With $m_{e}$ and $q_{e}$ the electron mass and charge, $v_{\|}^{e, i}$ the parallel collision frequency between ions and electrons, $v_{\nabla B}$ the vertical drift velocity and $\theta$ the vertical inclination of the magnetic field lines. In case of $\theta=0$, it is clear that the vertical electric field in the vessel cannot grow to infinity. At low $\theta, E_{z}$ may be defined by other short-circuiting mechanisms such as ion effects or currents flowing in the conducting limiters and the vacuum vessel that bound the RF plasma. The total conductance $\sigma_{\text {tot }}$ that limits the accumulation of charges in equation (5) is modified to

$$
\sigma_{\text {tot }}=\sigma_{\mathrm{o}}+\frac{q_{e}^{2}}{m_{e}} \frac{\sin ^{2} \theta}{v_{\|}^{e, i}}
$$

In the absence of $B_{z}$, the electric field is then given by

$$
E_{z, 3}=\frac{q_{e} v_{\nabla B}}{\sigma_{\mathrm{o}}} \propto \frac{T_{e}+T_{i}}{R B_{T} \sigma_{\mathrm{o}}}=f_{V(\text { 8.) }}\left(T_{e}+T_{i}\right)
$$

with $T_{i}$ the ion temperature. This scaling is currently implemented in the model, with tuneable numerical coefficient $f_{V(8 .)}$, and will be discussed in section 3 .

Finally, Van der Laan described the outward acceleration of a toroidal plasma column via a magnetohydrodynamic model, a microscopic model and energy calculations. These all yield the same formula for the outward acceleration [17]

$$
a_{r}=\frac{2 k}{m_{i} R}\left(T_{e}+Z T_{i}\right)
$$


with $k$ the Boltzman constant, $m_{i}$ the ion mass and $Z$ the ion charge number. Here, rather than considering a steady vertical electric field due to charge separation, the plasma is seen as practically neutral, and the outward motion results from a centrifugal force and a magnetic dipole force [17]. The case study in section 3 relates the outward acceleration to a drift velocity via the radial cross field mobility $\mu_{r}$ of the plasma. Together with the Einstein relation, this results into

$$
V_{r}=f_{V(9 .)} \frac{2 D_{r}}{R}
$$

with numerical coefficient $f_{V(\text { (9.) }}$. This corresponds, in case of Bohm-like diffusion and with $\sigma_{\mathrm{o}} \propto B_{T}^{-1}$, again to the scaling of equation (8).

2.2.3 Losses along field lines. The model includes equally spaced poloidal limiters at the low field side (LFS) and the high field side (HFS) boundaries of the radial simulation domain. The charged particle losses on these limiters are described in the model by a characteristic time in accordance to the Bohm criterion [18]

$$
\tau_{p}=\frac{2 \pi r}{N c_{s}}
$$

with $\mathrm{N}$ being the number of limiters and $\mathrm{c}_{\mathrm{s}}$ the ion sound velocity. The losses along the poloidal magnetic field component in the bulk plasma are described by eq. (3) and (4).

2.2.4 Neutrals. The transport of neutrals is approached by a diffusion process with coefficient

$$
D_{r, n}=\frac{1}{3} v_{c, n}\left(2 a^{-1}+\lambda_{n}^{-1}\right)^{-1} \lambda_{n}
$$

where $v_{c, n}$ and $\lambda_{n}$ are the collision frequency and the mean free path of the neutrals. In the case of low collisionality, this allows for a consistent estimation of the flux of neutrals to the walls similarly to equation (4). At high plasma densities, this allows producing the neutral pressure gradients that are expected in the case of fully ionised plasmas with electron temperatures above $T_{e} \gg 10 \mathrm{eV}$, as observed in ECRH plasmas in TCV.

\subsection{Edge conditions}

The plasma wall interaction (PWI) processes are represented in the model by particle and energy recycling coefficients. The reflection or recombination of particles at the wall surface is assumed to be immediate. Hence, the PWI description in the model assumes steady plasma and wall loading conditions. In the balance equations for neutral He and $\mathrm{H}_{2}\left(T_{\mathrm{He}}, T_{\mathrm{H}_{2}}<10 \mathrm{eV}\right)$, the particle reflection coefficient is set equal to 1 while the energy reflection coefficient is set below 0.1 , in close agreement with [19], [20]. The particle and energy reflection coefficients for hydrogen atoms is set at $R_{H}=0.5$ [19]. The remaining $\left(1-R_{H}\right)$ recombines on the wall surface to form $\mathrm{H}_{2}$ [21]. All ions are neutralised upon interaction with the surfaces, and recycle as $\mathrm{He}$ or $\mathrm{H}_{2}$. Finally, the $\mathrm{He}$ and $\mathrm{H}_{2}$ densities at the outer grid points are kept constant at the experimental neutral density readings as obtained by ionisation gauges.

\section{A case study for TCV helium ECRH plasmas}

ECRH plasmas are produced by coupling RF waves to electrons at the $1^{\text {st }}$ harmonic electron cyclotron resonance layer or at its $2^{\text {nd }}$ harmonic. Knowing the location at- and the species to which the power couples, makes these discharges exceptionally suitable for studying charged particle transport in toroidal magnetized plasmas. The plasma profiles at the HFS and LFS of the resonance layer result from the balance between transport and collisional processes only. This is in contrast to similar plasmas in the ion cyclotron range of frequencies where collisional absorption of RF power to both electrons and ions occurs all along the plasma radius.

Experiments in the TCV tokamak at EPFL in Switzerland assessed the applicability of ECRH discharges in helium at the second electron cyclotron (EC) harmonic to de-saturate the carbon-based plasma facing surfaces from deuterium [22]. The work was part of the preparations for the first operations campaign in JT-60SA. This section illustrates the utility of the reaction-diffusion-convection model through a case study reproducing 12 discharges from this particular experiment. $\operatorname{TCV}(R=0.88 \mathrm{~m}, a=0.25 \mathrm{~m})$ runs with gyrotrons at 82.7 $\mathrm{GHz}$ in X2 mode. The fraction $\eta_{e c}$ of the launched ECRH power $P_{e c}$ that couples to the plasma scales as $n_{e} T_{e}$ until refraction effects at densities above $2 \cdot 10^{19} \mathrm{~m}^{-3}$ significantly reduce the available power at the resonance layer [23]. Our 1D model includes the coupled ECRH power in the energy source term at the simulation grid point closest to the resonance layer:

$$
S_{E, e c}\left(r_{c e}, t\right)=\eta_{e c} P_{e c}
$$

Figure 1 and 2 show several radial density profiles measured in separate TCV discharges along an array of vertical interferometry chords. The displayed values are averaged over $0.5 \mathrm{~s}$, an error bar indicates the standard deviation of the signal. Figure 1 presents data of an ECRH power scan at fixed gas flow, magnetic field configuration and ECRH launch angle. When the ECRH power is increased, the maximum density increases approximately proportional in the considered density range. From this it is preliminary concluded that the advantage of better ECRH coupling at higher densities is compensated by other processes, as will be described in the next subsections. Two of the shown profiles have an identical ECRH power to demonstrate the discharge reproducibility (\#51514 and \#51518 at $180 \mathrm{~kW}$ ). Figure 2 presents data of a vertical magnetic field scan, while keeping the ECRH power and other parameters constant. An optimal, intermediate, vertical field strength exists that maximises the 


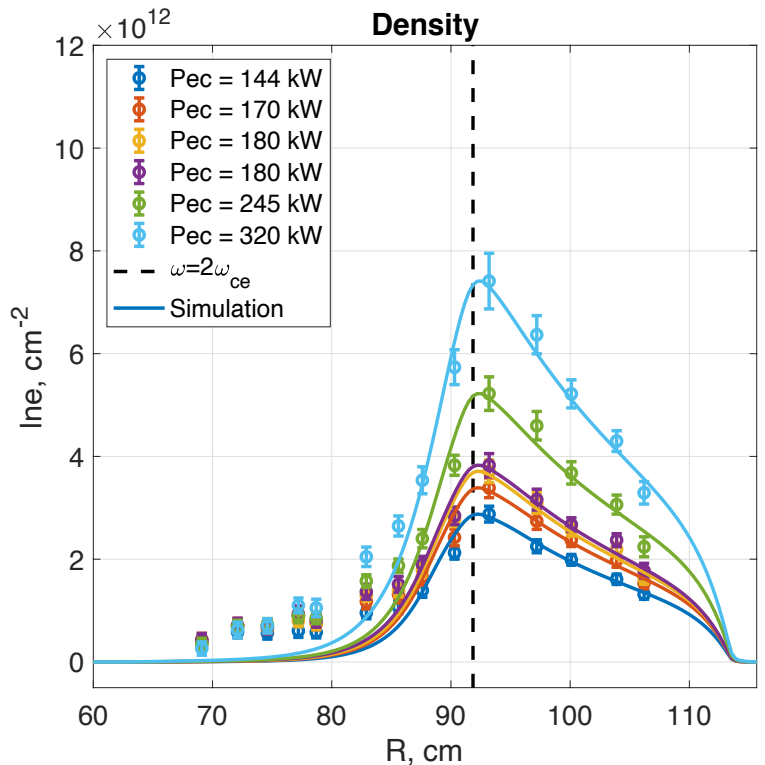

Figure 1: Effect of the launched ECRH power at $82.7 \mathrm{GHz}$ on the X2ECRH plasma density on TCV (\#51513-51519). The toroidal and poloidal injection angle were $-10.1^{\circ}$ and $4.6^{\circ}$ respectively, the vertical and radial magnetic field were $3.5 \mathrm{mT}$ and $0.5 \mathrm{mT}$ compared to toroidal field of $1.54 \mathrm{~T}$. The data points stand for time averaged experimental line integrated densities. Simulated profiles by Tomator-1D are given by solid lines.

density. It is often stated that the decrease in density at higher vertical magnetic field results from losses along the magnetic field lines. This will be discussed in next subsections. The shape of the plasma profiles is similar for all the discharges, with a density peak located just on the LFS of the resonance layer, a sharp density drop toward the HFS and an approximately linear decay toward the LFS, indicating no dramatic regime changes in transport, ionisation degree nor ECRH absorption. The solid lines are simulated density profiles. Overall, the model, with a detailed description of the collisional processes and conventional assumptions on the radial transport, is able to reproduce the experimental data very well. Small discrepancies observed at the HFS, e.g. at $B_{z}=0.25 \%$, may be due to collisional absorption of mode converted EC waves.

\subsection{Diffusion}

The numerical coefficient for diffusion, $f_{D}$ (eq. $3 \& 4$ ), used to simulate the experimental data set is plotted in figure $3 a$ and $3 \mathrm{~d}$. For the power scan, the coeffient is constant at $f_{D}=$ $0.081 \pm 3 \%$, about half the Spitzer constant. The coefficient in the vertical magnetic field scan is higher, $f_{D}=0.19 \pm$ $18 \%$, with an outlayer at $B_{z}=0.25 \%$ which is understood from the above-mentioned higher density at the HFS of the resonance layer in this discharge. The small standard deviation around the numerical coefficient shows that the implemented scaling of eq. (3) describes very well the changes in anomalous diffusion transport within the two scans. An

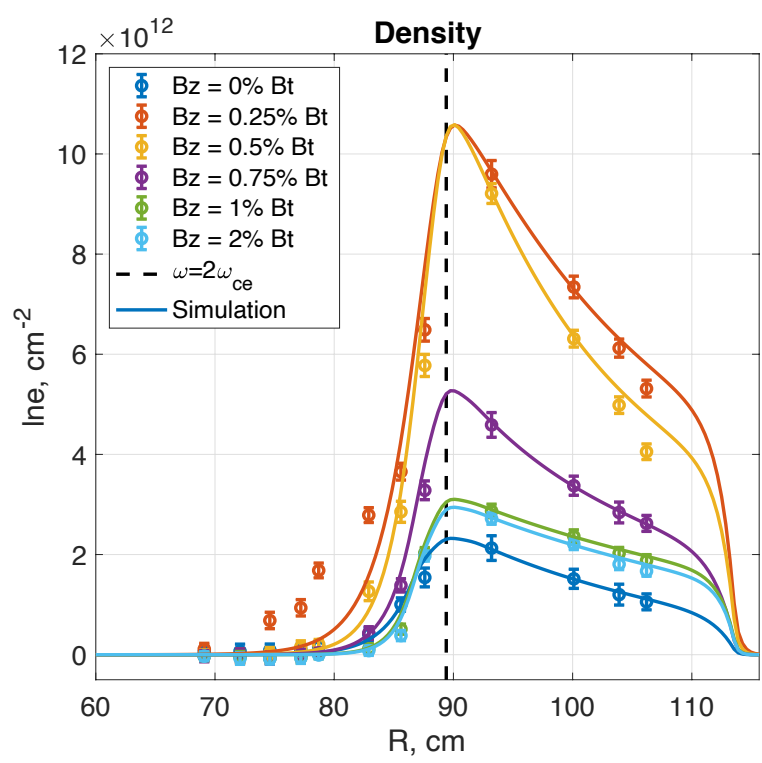

Figure 2: Effect of a vertical field on the X2-ECRH plasma density on TCV (\#57327-57335). The launched ECRH power was $400 \mathrm{~kW}$ at 82.7 $\mathrm{GHz}$ and $1.5 \mathrm{~T}$ with toroidal and poloidal injection angle of $19.3^{\circ}$ and $7.0^{\circ}$ respectively. The data points stand for time averaged experimental line integrated densities. Simulated profiles by Tomator-1D are given by solid lines.

improved experimental transport scaling can be proposed that reduces the difference between the scans, guiding further work on transport scalings for toroidal magnetized RF plasmas and future experiments. On the other hand, the different numerical coefficient may point out anomalies in the model assumptions. For example, the plasma temperature is on average lower in the vertical magnetic field scan. If (i) the model underestimates the plasma temperature, knowing eq. (3) is roughly proportional to the plasma temperature, or if (ii) the diffusion scales rather as $T^{n}$ with $n>1$, then indeed a lower $f_{D}$ is expected in the pulses with a vertical magnetic field.

\subsection{Convection}

The numerical coefficients $f_{V}$ (eq. 8 and 9) are plotted in figure $3 \mathrm{~b}$ and $3 \mathrm{e}$. The value is on average $f_{V(8 .)}=0.25 \pm 17 \%$ for the power scan, close to the Nakao estimate, while an upward trend is visible at rising power. Recalculating the coefficient as eq. (9) reduces the spread with $f_{V(9 .)}=6.5 \pm$ $12 \%$. A weaker dependency is seen on the vertical magnetic field. The outward convection stays approximately constant on applying a vertical magnetic field, at $f_{V(8 .)}=0.96 \pm 48 \%$, in contrast to what is expected based on eq. (6) in which the strength of the vertical electric field, resonsible for the outward $E \times B$ drift, rather reduces. This may indicate that the first term in eq. (7) is more important than the second in the studied range of vertical field values. Recalculating the coefficient as eq. (9), with $f_{V(\text { 9.) }}=10.9 \pm 23 \%$, both reduces the spread and approaches the numerical coeficient of the 

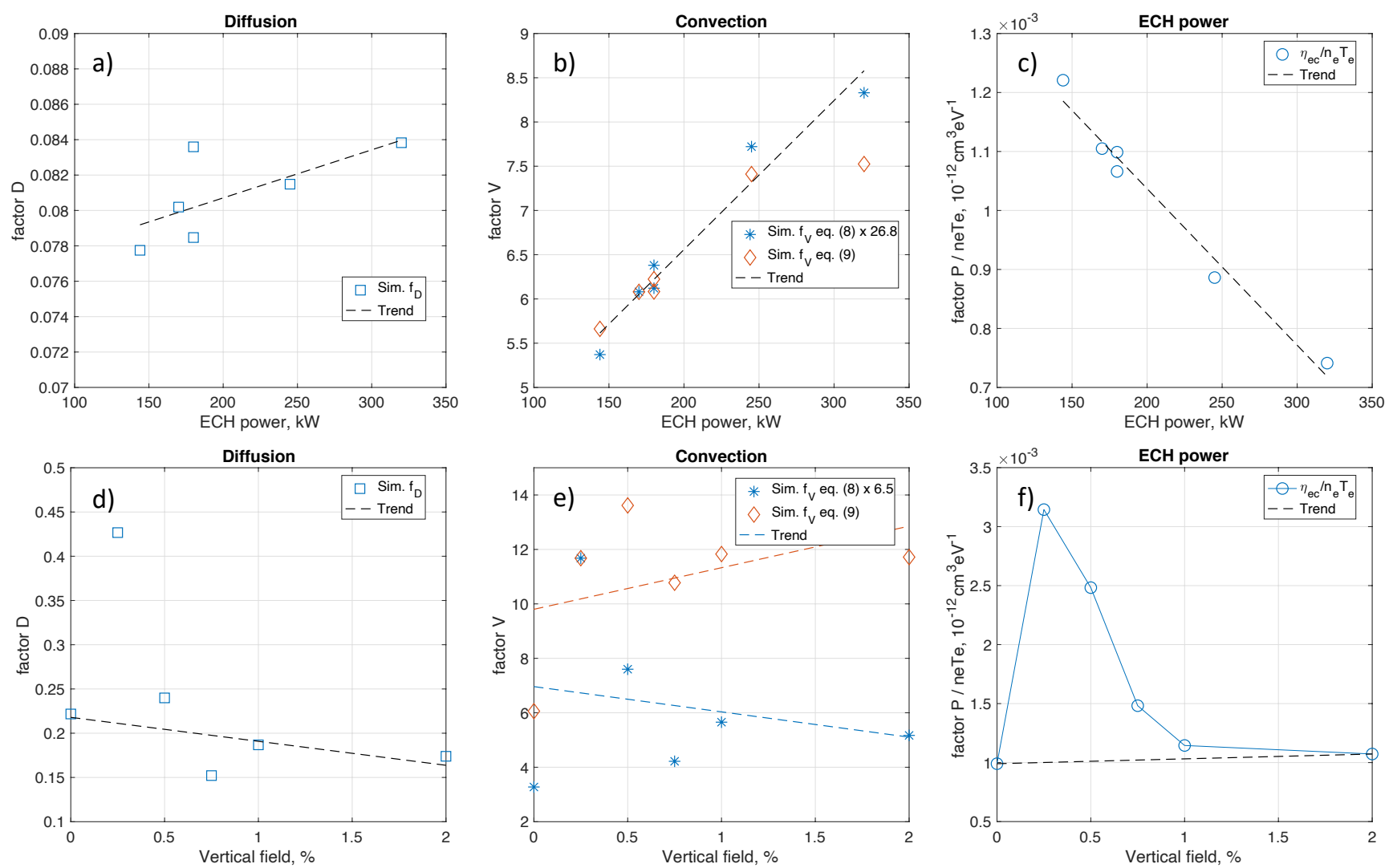

Figure 3: Overview of the numerical coefficients, $f_{D}(a, d), f_{V}(b, e)$ and $\eta_{e c}$, introduced in equations (3), (4), (8), (9) and (11), that are used to reproduce the profiles shown in figure $1(a-c)$ and $2(d-f)$.

power scan. The latter delivers a first evidence for the relation between the anomalous diffusion and the outward convection in toroidal plasmas (eq. 9).

\subsection{Absorption efficiency of launched ECRH power}

Figure $3 \mathrm{c}$ and $3 \mathrm{f}$ plots the ratio of the power absorption efficiency to the typical scaling for absorption of $\mathrm{X}$ waves at the $2^{\text {nd }}$ electron cyclotron harmonic, $\eta_{e c} / n_{e} T_{e}$. It is clearly seen that the absorption efficiency decreases at higher ECRH power compared to the $n_{e} T_{e}$ scaling. This trend, indicated with a dashed line in the plot, results from the power density of the launched EC bundle at the resonance layer. Farina described the maximum energy gain in $\mathrm{keV}$ of an electron at the $\mathrm{EC} 2^{\text {nd }}$ harmonic resonance as [24]

$$
W_{\text {max }} \cong 2.1 \frac{\sqrt{P_{e c}}}{w_{\|} f}
$$

with $P_{e c}$ being the launched EC power in MW, $w_{\|}$the width of the EC beam along the field lines in $\mathrm{m}$ and $f$ the wave frequency in GHz. Even for the discharge with the lowest power level in the scan of figure 1 one easily arrives at energies beyond $125 \mathrm{eV}$, i.e. the optimal energy for electron impact ionisation of helium [25]. Moving towards the discharge with $320 \mathrm{~kW}$, the tail in the electron energy distribution becomes too energetic for ionisation but also for thermalisation by coulomb collisions.

Similar values for $\eta_{e c} / n_{e} T_{e} \approx 10^{15} \mathrm{~cm}^{-3} \mathrm{eV}^{-1}$ are found in the vertical field scan, indicated by the dashed trend line, while a dramatic improvement is seen at intermediate vertical magnetic field values of $0.25-0.5 \%$. This may result firstly from a more optimal electron energy distribution. The vertical mean free path of $100 \mathrm{eV}$ electrons is estimated as $\lambda_{e} \sin \theta$ and can be 10 's of centimeters which effectively reduces the power density. Secondly, the possibility of improved absorption in these higher density discharges, e.g. by mode converted EC waves, needs to be investigated. Also the absolute absorption efficiency is maximum at intermediate vertical magnetic field values of $0.25-0.5 \%$. The absorption remains below $\eta_{e c}<25 \%$ in these pulses, leaving a significant amount of EC power as stray radiation.

\subsection{Comparison of terms in the particle balance}

Figure 4 compares the importance of the different terms in the particle balance (eq. 1). The plasma density at the HFS is determined mostly by the balance between inward diffusion of the charged particles that are produced by collisional processes in the area of the resonance layer, and outward convection. The latter is the main particle source at the LFS in the pulse with $B_{z}=0.5 \%$, and is balanced partly by losses 


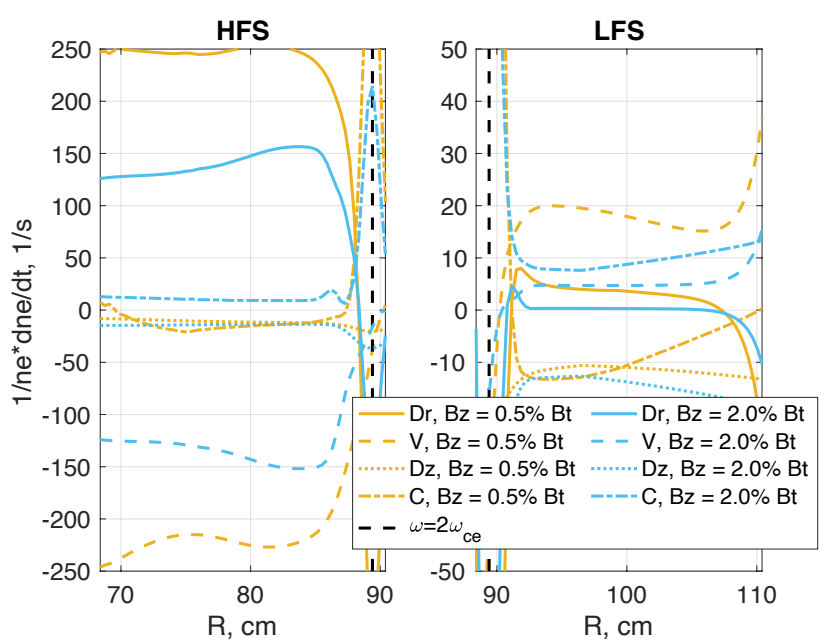

Figure 4: Contribution of radial diffusion $\left(D_{r}\right)$, convection (V) and losses along the vertical direction $\left(D_{z}\right)$ at the HFS (left) and LFS (right) of the resonance layer for two discharges with $400 \mathrm{~kW}$ of launched $X 2$ power at $82.7 \mathrm{GHz}$ and with respective vertical magnetic field of $0.5 \%$ and $2 \%$ of the toroidal magnetic field. The difference between the transport is balanced by collisional processes (C). The resonance layer location is shown by the black dashed line.

along the field lines and net recombination. At the higher field, $B_{z}=2.0 \%$, both net ionisation and convection provide density at the LFS. This is balanced mostly by losses along the field lines. It is therefore concluded that the beneficial effect of applying a small poloidal field to the ECRH plasma density indeed disappears due to losses along the field lines, as soon as the value is higher $\sim 0.5 \%$.

\section{Conclusion}

The 1-dimensional reaction-diffusion-convection code TOMATOR-1D describes plasma production by RF waves inside a tokamak using the Braginskii continuity and heat balance equations. The model simulates self-consistent radial density and temperature profiles for magnetised plasma mixtures of hydrogen and helium. It includes a detailed description of the collisional processes and is implemented with conventional assumptions on transport and EC power absorption. As such, the model reproduces the density profiles of X2 ECRH plasmas on TCV and provides better insight on the transport scalings and power absorption dependencies in parametric scans of the launched ECRH power and the applied vertical magnetic field.

A poloidal magnetic field dependent scaling for anomalous diffusion is proposed based on Bohm diffusion, with a numerical coefficient of the same order as the Bohm and Spitzer coefficients. Outward convection is implemented as to result from drifts in the toroidal magnetic field configuration. Two scalings are proposed, one where a steady vertical electric field by charge accumulation drives the outward $E / B$ $\mathrm{drift}$, and a second where the plasma is considered as a neutral body on which a net force acts, namely the centrifugal and magnetic dipole force on the gyrating particles. Relating the outward acceleration to a drift velocity via the radial cross field mobility reduces the spread on the numerical coefficients for convection that are used to reproduce the presented 12 TCV discharges. This provides a first evidence for the relation between the anomalous diffusion and the outward convection in toroidal plasmas.

The EC absorption efficiency in the TCV discharges, relative to the $n_{e} T_{e}$-scaling for $\mathrm{X} 2$ absorption, decreases at higher power. This is understood from the acceleration of electrons beyond the optimal energy for the electron impact ionisation of helium and provides experimental evidence of the theoretical estimates for the X2 EC wave-particle interaction [24]. Reducing the power density at the resonance layer may improve the electron energy distribution for better ionisation and thermalisation efficiency, which is indeed required to minimise the level of stray radiation. A dramatic increase of the absorption efficiency, compared to the $n_{e} T_{e^{-}}$ scaling for X2 absorption, is seen at intermediate vertical magnetic field values of $B_{z}=0.25-0.5 \% B_{T}$ and may result from both the effective decrease of the power density at the resonance layer or the additional absorption of mode converted EC waves in these higher density plasmas. The beneficial effect of applying a small poloidal field to the ECRH plasma density disappears due to losses along the field lines, as soon as the value is higher $\sim 0.5 \%$.

The model TOMATOR-1D provides a first tool to complement experimental data on RF plasma production with consistent model-based interpretations. To arrive at predictive capabilities for ECRH plasmas on JT-60SA and ITER, the proposed scalings, subsuming dependencies on the torus major radius and the toroidal field strength, need to be validated in a multi-machine study.

\section{Acknowledgements}

This work has been carried out within the framework of the EUROfusion Consortium and has received funding from the Euratom research and training programme 2014-2018 and 2019-2020 under grant agreement No 633053. The views and opinions expressed herein do not necessarily reflect those of the European Commission. This work was supported in part by the Swiss National Science Foundation.

\section{References}

[1] S. I. Braginskii, 'Transport Processes in a Plasma', Reviews of Plasma Physics, vol. 1, p. 205, 1965.

[2] D. Wünderlich, S. Dietrich, and U. Fantz, 'Application of a collisional radiative model to atomic hydrogen for diagnostic purposes', Journal of Quantitative Spectroscopy and Radiative Transfer, vol. 110, no. 1, pp. 62-71, Jan. 2009, doi: 10.1016/j.jqsrt.2008.09.015.

[3] Y. V. Ralchenko and Y. Maron, 'Accelerated recombination due to resonant deexcitation of metastable states', Journal of Quantitative Spectroscopy and Radiative Transfer, vol. 71, 
no. 2, pp. 609-621, Oct. 2001, doi: 10.1016/S00224073(01)00102-9.

[4] T. Wauters et al., 'OD model of magnetized hydrogenhelium wall conditioning plasmas', Plasma Phys. Control. Fusion, vol. 53, no. 12, p. 125003, Oct. 2011, doi: 10.1088/0741-3335/53/12/125003.

[5] H. H. Abou-Gabal and G. A. Emmert, 'Helium transport in vented divertors and limiters', Nucl. Fusion, vol. 31, no. 3, pp. 407-416, Mar. 1991, doi: 10.1088/0029-5515/31/3/001.

[6] P. Bachmann and D. Reiter, 'Kinetic Description of Elastic Processes in Hydrogen-Helium Plasmas', Contributions to Plasma Physics, vol. 35, no. 1, pp. 45-100, 1995, doi: 10.1002/ctpp.2150350106.

[7] P. Bachmann, D. Reiter, and A. K. Prinja, 'Simple models for evaluating the role of atomic processes in helium enrichment', Journal of Nuclear Materials, vol. 196-198, pp. 865-870, Dec. 1992, doi: 10.1016/S0022-3115(06)80158-3.

[8] R. Marchand, C. Illescas, X. Bonnin, and J. Botero, 'Radiative Losses and Electron Cooling Rates of Hydrogen, Helium, Carbon and Oxygen', IAEA NUCLEAR DATA 8ECTION, Vienna, INDC(NDS)-309, Jul. 1995. Accessed: Jul. 03, 2020. [Online]. Available: https://www.academia.edu/27468251/Radiative_Losses_an d_Electron_Cooling_Rates_of_Hydrogen_Helium_Carbon_a nd_Oxygen.

[9] D. Reiter, The Data File HYDHEL: Atomic and Molecular Data for EIRENE. Forschungszentrum Juelich $\mathrm{GmbH}, 2002$.

[10] O. Marchuk, G. Bertschinger, H.-J. Kunze, A. Urnov, and F. G. and, 'Recombination mechanisms in He-like argon spectra measured in low-density plasmas', J. Phys. B: At. Mol. Opt. Phys., vol. 40, no. 23, pp. 4403-4412, Nov. 2007, doi: 10.1088/0953-4075/40/23/001.

[11] V. P. Shevelko, D. Kato, M.-Y. Song, H. Tawara, I. Yu. Tolstikhina, and J.-S. Yoon, 'One-electron capture and target-ionization in He+-neutral-atom collisions', Nuclear Instruments and Methods in Physics Research Section B: Beam Interactions with Materials and Atoms, vol. 267, no. 20, pp. 3395-3402, Oct. 2009, doi: 10.1016/j.nimb.2009.07.021.

[12] R. Janev and J. J. Smith, 'Atomic and Plasma-Material Interaction Data for Fusion', J. Nucl. Fusion, no. 4, pp. 116121, 1993.

[13] M. B. Shah, P. McCallion, and H. B. Gilbody, 'Electron capture and ionisation in collisions of slow $\mathrm{H}+$ and $\mathrm{He} 2+$ ions with helium', J. Phys. B: At. Mol. Opt. Phys., vol. 22, no. 19, pp. 3037-3045, Oct. 1989, doi: 10.1088/09534075/22/19/018.

[14] S. Yoshikawa and D. J. Rose, 'Anomalous Diffusion of a Plasma across a Magnetic Field', The Physics of Fluids, vol. 5, no. 3, pp. 334-340, Mar. 1962, doi: 10.1063/1.1706619.

[15] S. Nakao, K. Ogura, Y. Terumichi, and S. Tanaka, 'Particle loss from an electron cyclotron resonance discharge plasma in the WT-2 device', Physics Letters A, vol. 96, no. 8, pp. 405408, Jul. 1983, doi: 10.1016/0375-9601(83)90325-0.

[16] S. H. Müller, A. Fasoli, B. Labit, M. McGrath, M. Podestà, and F. M. Poli, 'Effects of a Vertical Magnetic Field on Particle Confinement in a Magnetized Plasma Torus', Phys. Rev. Lett., vol. 93, no. 16, p. 165003, Oct. 2004, doi: 10.1103/PhysRevLett.93.165003.
[17] P. C. T. van der Laan, 'Drift of a plasma in a curved magnetic field', J. Nucl. Energy, Part C Plasma Phys., vol. 6, no. 6, pp. 559-566, Jan. 1964, doi: 10.1088/0368-3281/6/6/302.

[18] U. Bernhard, 'Transport Processes in the Plasma Edge', Fusion Science and Technology, vol. 53, no. 2T, pp. 229-242, Feb. 2008, doi: 10.13182/FST08-A1709.

[19] W. Eckstein, 'Calculated sputtering, reflection and range values', Max-Planck-Institut fuer Plasmaphysik, IPP--9/132, 2002. Accessed: Mar. 21, 2020. [Online]. Available: http://inis.iaea.org/Search/search.aspx?orig_q=RN:3305536 5.

[20] E. Vietzke, M. Wada, and M. Hennes, 'Reflection and adsorption of deuterium atoms and molecules on graphite', Journal of Nuclear Materials, vol. 266-269, pp. 324-329, Mar. 1999, doi: 10.1016/S0022-3115(98)00665-5.

[21] M. Rutigliano and M. Cacciatore, 'Isotope and surface temperature effects for hydrogen recombination on a graphite surface', Chemphyschem, vol. 9, no. 1, pp. 171181, Jan. 2008, doi: 10.1002/cphc.200700394.

[22] D. Douai et al., 'Development of helium electron cyclotron wall conditioning on TCV', Nucl. Fusion, vol. 58, no. 2, p. 026018, Dec. 2017, doi: 10.1088/1741-4326/aa9d2b.

[23] D. Farina, 'A Quasi-Optical Beam-Tracing Code for Electron Cyclotron Absorption and Current Drive: GRAY', Fusion Science and Technology, vol. 52, no. 2, pp. 154-160, Aug. 2007, doi: 10.13182/FST07-A1494.

[24] D. Farina, 'Nonlinear collisionless electron cyclotron interaction in the pre-ionisation stage', Nucl. Fusion, vol. 58, no. 6, p. 066012, Apr. 2018, doi: 10.1088/1741$4326 /$ aabaa7.

[25] A. A. Sorokin, I. L. Beigman, S. V. Bobashev, M. Richter, and L. A. Vainshtein, 'Total electron-impact ionization cross sections of helium', J. Phys. B: At. Mol. Opt. Phys., vol. 37, no. 15 , pp. 3215-3226, Jul. 2004, doi: 10.1088/09534075/37/15/015. 